\title{
Aktuelle Reformvorschläge zum Einkommensteuertarif
}

\section{Mittelschichten entlasten, hohe Einkommen belasten}

\begin{abstract}
Der aktuelle Einkommensteuertarif belastet Mittelschichten und Besserverdienende relativ stark durch den schnellen Anstieg der Progression. Entlastungen durch eine Anhebung des Grundfreibetrags oder eine Abmagerung des Mittelstandsbauchs führen leicht zu Steuerausfällen von höheren zweistelligen Milliardenbeträgen im Jahr. Erhöhungen der Spitzensteuersätze wirken dem entgegen, allerdings sind die Aufkommenspotenziale bei den Hochverdienenden begrenzt. Sollen zum Beispiel die unteren $95 \%$ der Bevölkerung um $1 \%$ ihres steuerpflichtigen Einkommens entlastet werden, muss man die obersten $5 \%$ mit knapp $3 \%$ belasten, die Grenzbelastungen müssen um 5,5 Prozentpunkte steigen.
\end{abstract}

Der Einkommensteuertarif steht regelmäßig im Zentrum steuerpolitischer Debatten - seit Mitte des 19. Jahrhunderts die modernen Einkommensteuersysteme entstanden sind (Buggeln, 2018). Das zentrale Moment der Einkommensteuer ist ihre Bemessung nach der „persönlichen Leistungsfähigkeit“. Es werden alle Einkommensquellen zusammengefasst und davon Vorsorgeaufwendungen, weitere Sonderausgaben oder Familienunterhalt abgezogen. Die personenbezogene Einkommensermittlung ermöglicht eine progressive Steuerbelastung, also steigende Steuersätze bei höherem Einkommen. Ferner kann die Steuerbelastung nach weiteren sozio-ökonomischen Verhältnissen differenziert werden - Familienstand und Kinder, Alter, Einkommensarten, Behinderungen oder Krankheiten. Eine entsprechende nach persönlichen Merkmalen differenzierte Steuerlastverteilung lässt sich nur sehr unvollkommen mit den indirekten Konsumsteuern oder Objektsteuern auf Vermögen und Ertragsquellen, die bis heute einen erheblichen Teil des Steueraufkommens ausmachen, abbilden. Daher galt und gilt die Einkommensteuer als „Königin der Steuern“

(C) Der/die Autor:in(nen) 2021. Open Access: Dieser Artikel wird unter der Creative Commons Namensnennung 4.0 International Lizenz veröffentlicht (creativecommons.org/licenses/by/4.0/deed.de).

Open Access wird durch die ZBW - Leibniz-Informationszentrum Wirtschaft gefördert.

Dr. Stefan Bach ist wissenschaftlicher Mitarbeiter am Deutschen Institut für Wirtschaftsforschung (DIW Berlin).
(Popitz, 1926), als „die reinste - und technisch und juristisch schönste - Gestalt des Steuergedankens überhaupt“, „die beste Leistung und der Höhepunkt der Steuerkunst des liberalen Bürgertums“" (Schumpeter, 1929).

Namentlich der progressive Einkommensteuertarif bewegt die Gemüter seit jeher. Er löst gleichermaßen politische und ideologische Ambitionen wie Bedenken aus. Sozialkonservative, Linksliberale und Linke sehen in der Steuerprogression die Möglichkeit, die „starken Schultern“ höher zu belasten, damit den Wohlfahrtsstaat zu finanzieren und die hohen Abgabenbelastungen der Mittelschichten zu senken. (Neo-)Liberale und Liberalkonservative fürchten zumeist die negativen Wirkungen hoher Steuersätze auf Leistungsanreize oder die Wirtschafts- und Eigentumsordnung.

Progressive Einkommensteuer belastet nur gut die Hälfte des Volkseinkommens

Tatsächlich ist die progressive Einkommensteuer nur ein Pfeiler des zeitgenössischen Steuersystems. Lohnsteuer und veranlagte Einkommensteuer machten vor der Corona-Krise (2019) etwa $38 \%$ des gesamten Steueraufkommens aus (vgl. Abbildung 1). Auf die nicht veranlagten Kapitalertragsteuern und die Unternehmensteuern entfielen in den vergangenen Jahren knapp $15 \%$ des Steueraufkommens. Diese belasten zwar überwiegend Haushalte mit hohen Einkommen, verwenden aber proportionale Tarife und sind nicht (mehr) in die progressive Einkommensteuer eingebunden. Der Rest des Steueraufkommens entfällt auf Mehrwertsteuer, Verbrauchsteuern (auf Energie, Tabak, Alkoholika etc.), Grundsteuern und die übrigen Objekt- und Aufwandsteuern. Diese belasten überwie- 
Abbildung 1

Steuerpflichtige Einkommen sowie Lohn- und Einkommensteuer 1960 bis 2019

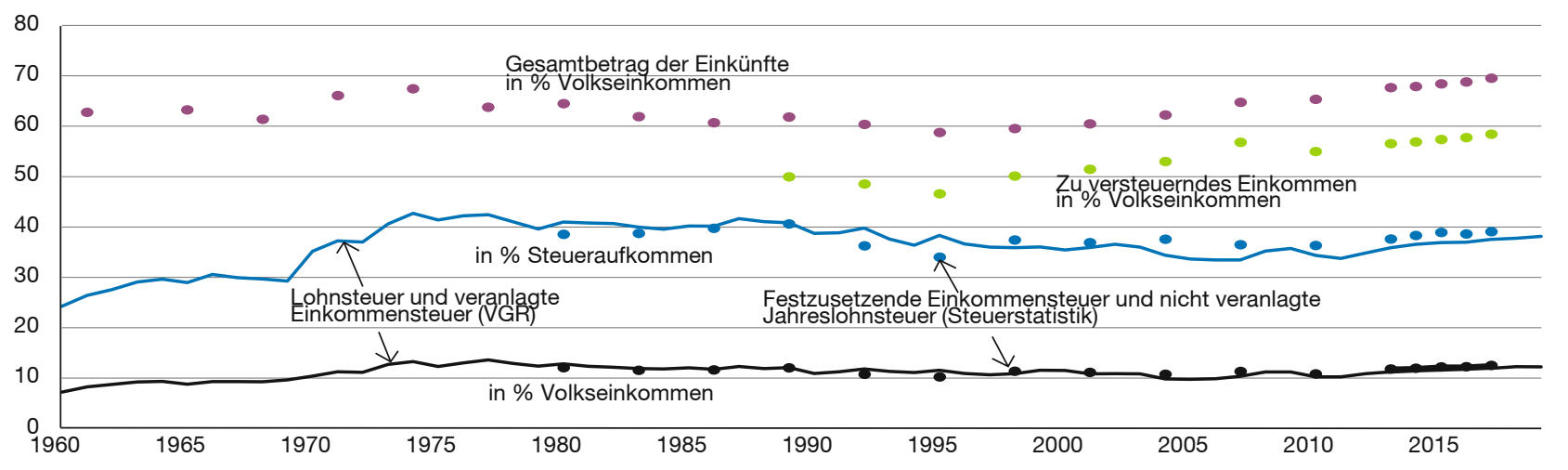

Quellen: Statistisches Bundesamt, Lohn- und Einkommenstatistik (Dreijähriger Rhythmus 1965 bis 2013); Volkswirtschaftliche Gesamtrechnungen (VGR).

gend den Verbrauch und wirken daher zumeist regressiv auf das laufende Periodeneinkommen - sie treffen also Haushalte mit niedrigen Einkommen relativ stärker als Haushalte mit höheren Einkommen. Daher ist die gesamte Steuerlastverteilung deutlich weniger progressiv als die Einkommensteuer selbst (Bach, Beznoska und Steiner, 2016). Man kann es auch so interpretieren: Die Progressivität der Einkommensteuer gleicht die Regressivität der Verbrauchsteuern aus.

Die in der Lohn- und Einkommensteuerstatistik erfassten steuerpflichtigen Bruttoeinkommen (Gesamtbetrag der Einkünfte) beliefen sich in der letzten verfügbaren Welle für 2017 (Statistisches Bundesamt, 2020) auf knapp 70\% des Volkseinkommens der Volkswirtschaftlichen Gesamtrechnungen (VGR), also der Summe aus Arbeitseinkommen sowie Unternehmens- und Vermögenseinkommen. Maßgebliche Gründe für diese Untererfassung sind die Steuerfreiheit der Arbeitgeberbeiträge zur Sozialversicherung, die nicht (mehr) in die Veranlagung zur Einkommensteuer integrierten Unternehmensgewinne und Kapitaleinkommen sowie steuerfreie Einkünfte, etwa Minijobs, befreite Auslandseinkünfte oder Vermögenseinkommen gemeinnütziger Institutionen.

In den 1980er und 1990er Jahren war die Erfassungsquote deutlich geringer, obgleich die Unternehmens- und Vermögenseinkünfte damals stärker in die persönliche Einkommensteuer einbezogen waren. Dies dürfte vor allem an den seinerzeit ausgeprägten Steuervergünstigungen und Gestaltungsmöglichkeiten bei den Unternehmensund Vermietungseinkünften gelegen haben. Diese sind durch die Steuerreformen ab Mitte der 1990er Jahre deutlich reduziert worden. Insoweit hat die „Tarifwahrheit“ der Einkommensteuer zugenommen (Esser, 2009). Allerdings werden Alterseinkünfte mit dem Übergang zur nachgelagerten Besteuerung zunehmend steuerpflichtig, die als Transfereinkommen nicht im Volkseinkommen der VGR enthalten sind. Nach Abzug der Sonderausgaben und auBergewöhnlichen Belastungen sowie der Kinderfreibeträge ergibt sich das zu versteuernde Einkommen, das zuletzt (2017) etwa $58 \%$ des Volkseinkommens ausmachte. Dieses ist die Basis für den Einkommensteuertarif. Nach Abzug des Grundfreibetrags ergibt sich für 2017 ein Einkommensaggregat von $41 \%$ des Volkseinkommens, das effektiv vom Einkommensteuertarif belastet wird.

\section{Schneller Belastungsanstieg durch Steuerprogression}

Die tarifliche Einkommensteuer ist in $\S 32$ a EStG definiert als abschnittweise Funktion des zu versteuernden Einkommens. Beim Steuertarif 2021 bleiben Einkommen bis zur Höhe des Grundfreibetrags von 9.744 Euro im Jahr unbesteuert, der das Existenzminimum repräsentiert. In den anschließenden beiden Progressionszonen werden quadratische Funktionen verwendet. Somit verlaufen die Grenzsteuersätze linear, also die Steuerbelastungen des nächsten steuerpflichtigen Euro (vgl. Abbildung 2). Daher spricht man auch von linear-progressiven Tarifen. Ab dem Eingangsteuersatz von $14 \%$ steigen die Grenzbelastungen zunächst steil an und erreichen bei einem zu versteuernden Einkommen von 14.753 Euro bereits knapp $24 \%$. In der anschließenden zweiten Progressionszone steigen die Grenzsteuersätze deutlich langsamer, sodass beim Übergang ein Knick entsteht. Der erste Spitzensteuersatz von $42 \%$ wird bei knapp 58.000 Euro erreicht. Für Einkommen ab 275.000 Euro steigt der Spitzensteuersatz auf $45 \%$, das ist der sogenannte Reichensteuerzuschlag. Ab 2021 wird der Solidaritätszuschlag erst oberhalb einer Freigrenze von 16.956 Euro Einkommensteuerbelastung erhoben, was einem zu versteuernden Einkommen von 62.200 Euro entspricht. Die anschließende Gleitzone reduziert die Grenzbelastung des Solidaritätszuschlags auf $11,9 \%$ des Grenzsteuersatzes der Einkommensteuer (§ 3 Abs. 3 SolzG). Dadurch steigt die gesamte Grenzbelastung durch Einkommensteuer und So- 
lidaritätszuschlag auf knapp $47 \%$ bis zum Ende der Gleitzone bei 97.000 Euro zu versteuerndem Einkommen.

Die Steuerprogression wird üblicherweise an den Durchschnittsteuersätzen gemessen, also der Steuerbelastung bezogen auf das steuerpflichtige Einkommen (Pollak, 1980). Sie entsteht zum einen durch den Grundfreibetrag (indirekte Progression), bezogen auf das vorgelagerte Bruttoeinkommen vor Abzügen auch durch Sonderausgaben, außergewöhnliche Belastungen und weitere Freibeträge. Zum anderen entsteht sie durch steigende Grenzsteuersätze auf steigende zu versteuernde Einkommen (direkte Progression). Dadurch verlaufen die Durchschnittsteuersätze bei niedrigen Einkommen deutlich unterhalb der Grenzsteuersätze. Bei hohen Einkommen nähern sie sich dem Spitzensteuersatz immer weiter an. Die Unterscheidung zwischen den Grenzsteuersätzen bezogen auf einen zusätzlich verdienten Euro und den Durchschnittsteuersätzen auf das gesamte steuerpflichtige Einkommen ist wesentlich für das Verständnis des progressiven Steuertarifs und seiner technischen Mechanik. Da dies in den steuerpolitischen Diskursen häufig nicht unterschieden wird, können leicht Missverständnisse entstehen.

\section{Linear-progressiver Steuertarif - zurück zu Stoltenberg}

Der schnelle Anstieg der Steuerbelastung im Eingangsbereich des Einkommensteuertarifs und der damit verbundene Mittelstandsbauch werden oft kritisiert. In der ersten kurzen Progressionszone steigen die Grenzbelastungen bis zur Knickstelle steil an, die Durchschnittsbelastungen erhöhen sich schnell. Im Zusammenwirken mit den Sozialbeiträgen entstehen hohe Grenzbelastungen auf eigenes Einkommen. Bei Niedrigverdienenden kommt der Abbau von Transferleistungen beim Bezug von Grundsicherung, Kinderzuschlag oder Wohngeld hinzu. Diese hohen Grenzbelastungen gelten als leistungsfeindlich und lösen Steuervermeidung aus. Es entstehen hohe Steuer- und Abgabenbelastungen bei den Mittelschichten und Besserverdienenden, also im 5. bis zum 8. Dezil der Einkommensverteilung (vgl. Tabelle 2). Dies gilt vor allem für Alleinstehende ohne Kinder, während Verheiratete und Familien mit Kindern vom Ehegattensplitting und vom Familienleistungsausgleich profitieren (OECD, 2021). Hochverdienende wurden über die letzten Jahrzehnte deutlich entlastet - bei den Spitzensteuersätzen, aber auch bei Unternehmensteuern, Kapitalertragsteuern und Vermögensteuer.

Seit Jahren gibt es eine Entlastungsdiskussion für geringe und mittlere Einkommen. Viele Reformvorschläge wollen den Mittelstandsbauch abmagern (Bach und Buslei, 2017). Dies legt nahe, zu einer einheitlichen linear-progressiven Tariffunktion überzugehen, wie sie ab 1990 unter der Ägide des Bundesfinanzministers Gerhard Stoltenberg eingeführt wurde. Die linear-progressive Tariffunktion hat eine
Abbildung 2

Einkommensteuertarif und Solidaritätszuschlag, Recht 2021

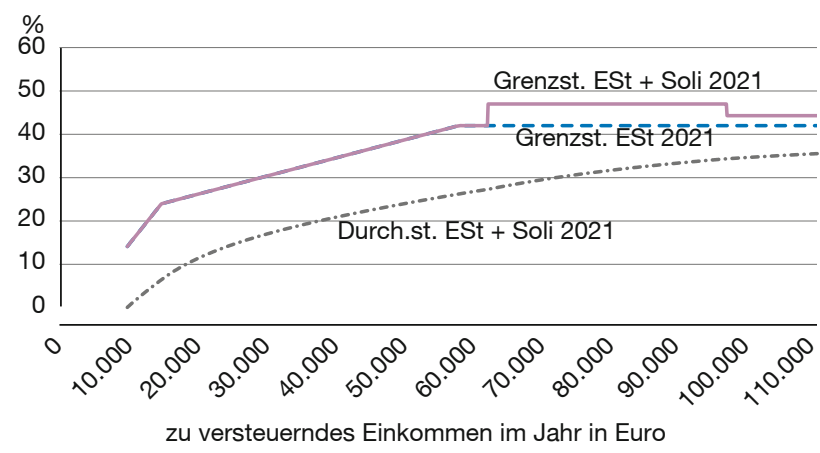

Quelle: eigene Berechnungen.

gewisse tarifästhetische und steuerpolitische Anmut: Der unschöne Knick wird vermieden, die Grenzbelastungen steigen kontinuierlich an. Auf Grundlage der Tarifeckwerte des Tarifs 2021 wird in Abbildung 3 als erstes Szenario eine solche Tariffunktion mit linear steigenden Grenzsteuersätzen von $14 \%$ bis $42 \%$ verwendet. In Tabelle 1 sind die Aufkommens- und Verteilungswirkungen dieser und der im Folgenden diskutierten Tarifreformen dargestellt. Sie werden mit fortgeschriebenen Daten der Lohn- und Einkommensteuerstatistik 2016 simuliert. Die Einkommensverteilung bezieht sich auf die gesamte erwachsene Bevölkerung, zusammen veranlagte Ehepaare werden als zwei Personen gezählt. Nicht lohnsteuerpflichtige oder nicht zur Einkommensteuer veranlagte Personen werden in der untersten Einkommensgruppe berücksichtigt. Berechnet werden die unmittelbaren Erstrundeneffekte der Reformen auf Steueraufkommen und Verteilung. Anpassungen der Steuerpflichtigen und weitere wirtschaftliche Wirkungen werden vernachlässigt.

Eine solche linear-progressive Stoltenberg-Tarifreform bedeutet Entlastungen und damit Steuerausfälle in Höhe von 39 Mrd. Euro - das sind $11 \%$ des Einkommensteueraufkommens, $4,5 \%$ des Steueraufkommens oder gut $1 \%$ des Bruttoinlandsprodukts. In Relation zum zu versteuernden Einkommen profitieren die oberen Mittelschichten und die Besserverdienenden bis zum 9. Dezil der Einkommensverteilung am stärksten. In absoluten Euro-Beträgen werden die Besser- und Hochverdiener am stärksten entlastet, denn sie profitieren von der Tarifsenkung über den gesamten Einkommensbereich, was sich an der Fläche zwischen den Grenzsteuersatzkurven ablesen lässt. Dadurch steigt die Entlastung bei der Einkommensteuer für einen einzelveranlagten Steuerpflichtigen auf maximal 1.700 Euro im Jahr, die ab der Einkommensgrenze des ersten Spitzensteuersatzes erreicht wird. Einschließlich des Rest-Solidaritätszuschlags steigt die Entlastung auf 1.900 Euro bei steuerpflichtigen Einkommen innerhalb der Milderungszone und auf 1.795 Euro bei Einkommen darüber. 
Abbildung 3

Einkommensteuertarif und Solidaritätszuschlag, Recht 2021 und sechs Reformszenarien

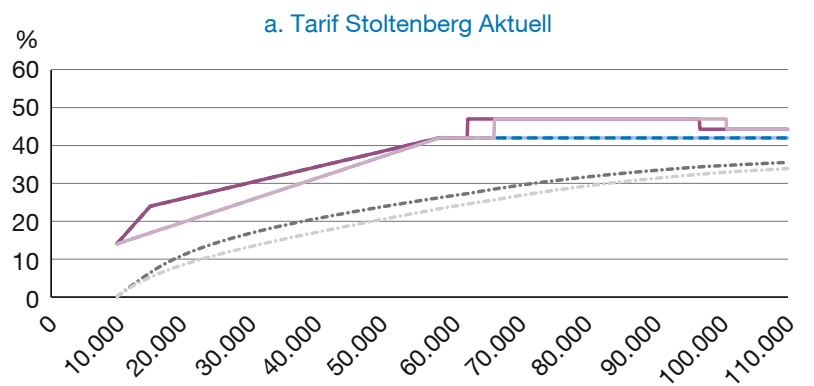

zu versteuerndes Einkommen im Jahr in Euro

- - - - Grenzst. ESt 2021

- - - - Grenzst. ESt Tarif Stoltenberg Aktuell

— Grenzst. ESt + Soli 2021

_ Grenzst. ESt + Soli Tarif Stoltenberg Aktuell

- - - - Durch.st. ESt + Soli 2021

Durch.st. ESt + Soli Tarif Stoltenberg Aktuell

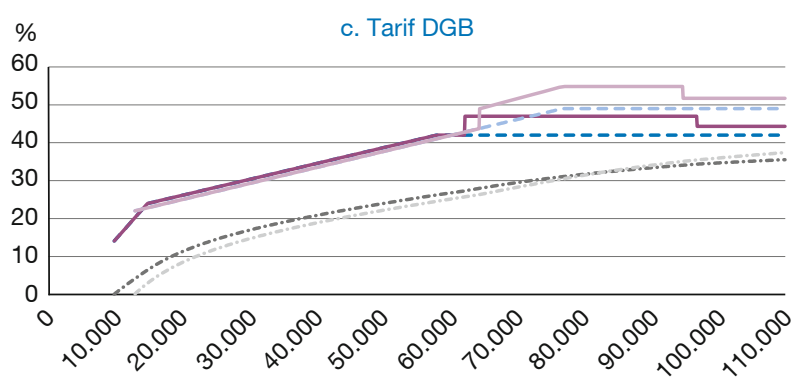

zu versteuerndes Einkommen im Jahr in Euro

$$
\begin{array}{ll}
- \text { - - - Grenzst. ESt } 2021 \\
- \text { - Grenzst. ESt Tarif DGB } \\
\hline \text { Grenzst. ESt + Soli } 2021 \\
\hline \text { - - - - Gurenzst. ESt + Soli Tarif DGB } \\
\hline- \text { Drcht. ESt + Soli } 2021
\end{array}
$$$$
\text { Durch.st. ESt + Soli Tarif DGB }
$$

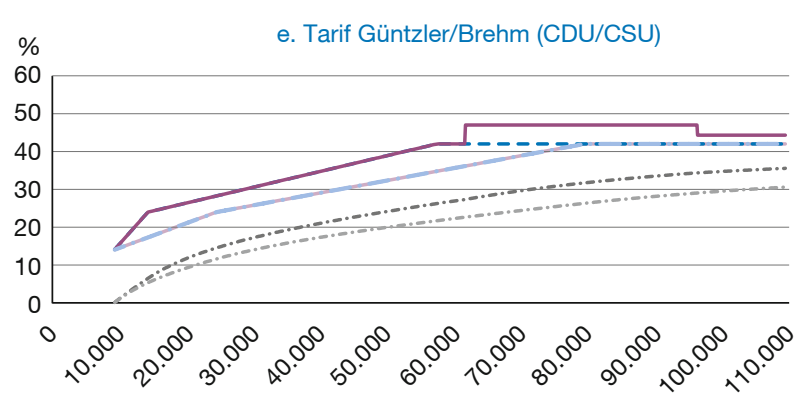

zu versteuerndes Einkommen im Jahr in Euro

- - - - Grenzst. ESt 2021

- - - - Grenzst. ESt Tarif Güntzler/Brehm

- Grenzst. ESt + Soli 2021

_ Grenzst. ESt + Soli Tarif Güntzler/Brehm

- - - - Durch.st. Est + Soli 2021

Durch.st. ESt + Soli Tarif Güntzler/Brehm

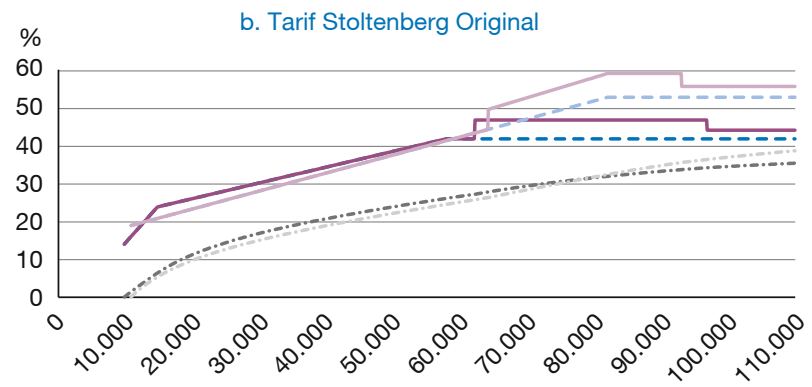

zu versteuerndes Einkommen im Jahr in Euro

- - - - Grenzst. ESt 2021

- - - = Grenzst. ESt Tarif Stoltenberg Original

- Grenzst. ESt + Soli 2021

— Grenzst. ESt + Soli Tarif Stoltenberg Original

- - - - Durch.st. ESt + Soli 2021

Durch.st. ESt + Soli Tarif Stoltenberg Original

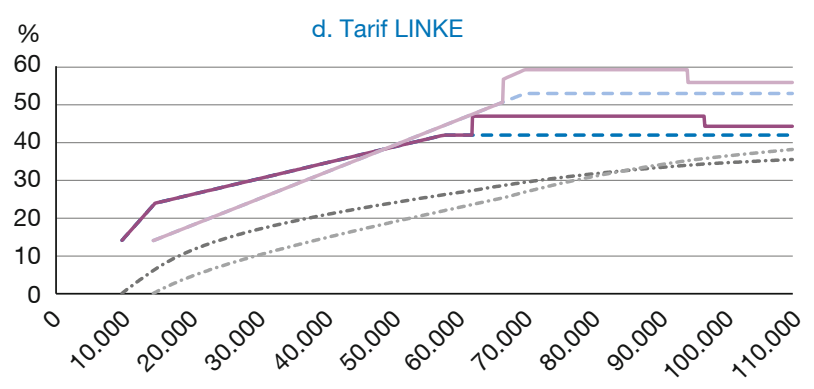

zu versteuerndes Einkommen im Jahr in Euro

\section{- - - - Grenzst. ESt 2021}

- - - - Grenzst. ESt Tarif LINKE

Grenzst. ESt + Soli 2021

Grenzst. ESt + Soli Tarif LINKE

- - - - Durch.st. ESt + Soli 2021

Durch.st. ESt + Soli Tarif LINKE

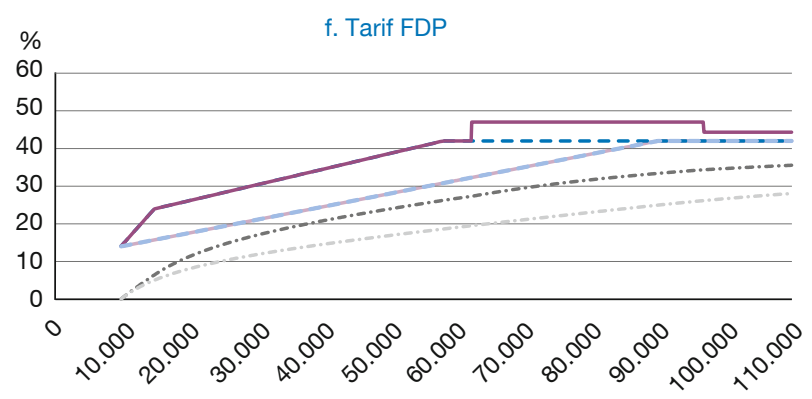

zu versteuerndes Einkommen im Jahr in Euro

- - - - Grenzst. ESt 2021

- - - Grenzst. ESt Tarif FDP

- Grenzst. ESt + Soli 2021

- Grenzst. ESt + Soli Tarif FDP

- - - - Durch.st. ESt + Soli 2021

Durch.st. ESt + Soli Tarif FDP

Quellen: eigene Berechnungen auf Grundlage des Einkommensteuertarifs 2021 und der Reformszenarien. 
Will man die Steuermindereinnahmen einer solchen Reform im Einkommensteuertarif reduzieren, muss man den Eingangsteuersatz des Tarifs oder den Spitzensteuersatz deutlich erhöhen. Hierzu wird in Abbildung 3 eine aufkommensneutrale Stoltenberg-Tarifreform mit den originalen Grenzsteuersatz-Eckwerten des Einkommensteuertarifs 1990 entwickelt: Eingangsteuersatz 19\% und Spitzensteuersatz $53 \%$. Der relativ niedrige Eingangsteuersatz von $14 \%$ ist bei den Steuerreformen der rot-grünen Bundesregierung Anfang der Nullerjahre eingeführt worden. Mit Blick auf die seinerzeitigen Arbeitsmarkt- und Sozialreformen sollte damit ein Signal für Niedrigverdienende gesetzt werden. Durch den steilen Anstieg der Grenzsteuersätze im Eingangsbereich betrifft das aber nur wenige Steuerpflichtige, ist also eher Symbolik ohne große wirtschaftliche Wirkungen. Außerdem war der Spitzensteuersatz auf $42 \%$ gesenkt worden, um bei Besser- und Spitzenverdienern die Leistungsanreize zu erhöhen und die Gestaltungsanreize zu verringern. Ab 2007 wurde dann der Reichensteuerzuschlag für sehr hohe Einkommen eingeführt.

Um Geringverdiener nicht durch die Erhöhung des Eingangsteuersatzes zu belasten, wird für den Stoltenberg-Originaltarif der Grundfreibetrag um 1.000 Euro auf 10.744 Euro zu versteuerndes Einkommen erhöht. Die Einkommensgrenze des Spitzensteuersatzes wird auf 82.000 Euro zu versteuerndes Einkommen erhöht, gegenüber knapp 58.000 Euro beim gegenwärtigen Tarif für den ersten Spitzensteuersatz. Dadurch verläuft der Anstieg der Grenzsteuersätze im Vergleich zur zweiten Progressionszone des gegenwärtigen Tarifs etwas steiler, der Grenzsteuersatz von $42 \%$ wird bei 59.000 Euro erreicht. Einschließlich Rest-Solidaritätszuschlag werden einzelveranlagte Steuerpflichtige bis zu einem zu versteuernden Einkommen von 78.600 Euro entlastet, bei zusammenveranlagten Paaren bis zum doppelten Einkommen. Diese Tarifreform geht in etwa aufkommensneutral auf - in ihren kurzfristigen Erstrundeneffekten, ohne Berücksichtigung weiterer wirtschaftlicher Wirkungen. Auf den Reichensteuerzuschlag von 3 Prozentpunkten Grenzsteuersatz bei hohen Einkommen wird hier verzichtet. Dieser würde gut 2 Mrd. Euro Mehreinnahmen erzielen. Auch bei dieser Reform werden die Mittelschichten und die Besserverdienenden bis zum $95 \%$-Perzentil der Einkommensverteilung entlastet, um etwa 18 Mrd. Euro im Jahr. Die Hochverdiener darüber werden dagegen deutlich belastet durch den Anstieg der Spitzensteuersätze. Im obersten Perzentil der Einkommensverteilung steigen die Einkommensteuerbelastungen um 7,6\%.

Wenn man die Entlastungen stärker auf die Geringverdiener im Eingangsbereich des Steuertarifs konzentrieren will, bietet sich eine deutlichere Erhöhung des Grundfreibetrags an. Um die damit verbundenen Steuerausfälle zu kompensieren, muss man dann den Eingangsteuersatz oder die Spitzensteuersätze stärker erhöhen. So schlägt der
Deutsche Gewerkschaftsbund (DGB) eine Erhöhung des Grundfreibetrags um gut 3.000 Euro auf 12.800 Euro vor (Deutscher Gewerkschaftsbund, 2021). Der Eingangsteuersatz soll $22 \%$ betragen und einen ersten Spitzensteuersatz von $49 \%$ ab einem zu versteuernden Einkommen von 76.800 Euro erreichen. Ab 130.000 Euro ist ein Reichensteuerzuschlag von $52 \%$ vorgesehen. Dadurch werden Geringverdiener und untere Mittelschichten stärker entlastet als bei der aufkommensneutralen Stoltenberg-Reform. Auch die Besserverdiener werden etwas stärker entlastet, die Belastungsgrenze liegt bei 83.000 Euro des zu versteuernden Einkommens. Da die Hochverdiener nicht so stark belastet werden wie beim Stoltenberg-Originaltarif, bedeutet die Reform Mindereinnahmen von gut 12 Mrd. im Jahr.

Auch SPD, Grüne und Linke wollen mit ihren aktuellen Vorschlägen zum Einkommensteuertarif vor allem den Grundfreibetrag erhöhen und die Spitzensteuersätze erhöhen (Beznoska und Hentze, 2021). Am weitesten geht hier die Linke: Grundfreibetrag 14.400 Euro, linear-progressiver Tarifverlauf bis $53 \%$ ab 70.000 Euro zu versteuerndes Einkommen, Reichensteuersatz $60 \%$ und ein zweiter Reichensteuersatz von $75 \%$ ab 1 Mio. Euro zu versteuerndes Einkommen. Eine derart starke Erhöhung des Grundfreibetrags führt in Kombination mit dem linear-progressiven Stoltenberg-Tarif zu Entlastungen von $67 \mathrm{Mrd}$. Euro im Jahr für die unteren $95 \%$ der Bevölkerung, die auch durch die kräftigen Spitzensteuersatz-Erhöhungen nur zum geringen Teil ausgeglichen werden. Daher führt die Reform zu Mindereinnahmen von 46 Mrd. Euro im Jahr. Union und FDP fürchten höhere Spitzensteuersätze wie der Teufel das Weihwasser. Kompromissbereitschaft signalisiert man bei einer Abschaffung des Rest-Solidaritätszuschlags, die durch eine Erhöhung der Spitzensteuersätze kompensiert werden kann. Zugleich wollen die Steuerpolitiker:innen dieser Parteien meist Mittelschichten und Besserverdienende kräftig entlasten durch eine Abmagerung des Mittelstandsbauchs und die Erhöhung der Einkommensgrenze, ab der der erste Spitzensteuersatz einsetzt. Dies bedeutet hohe Mindereinnahmen bei der Einkommensteuer.

Ein Beispiel hierfür ist der Vorschlag der Unions-Abgeordneten Fritz Güntzler und Sebastian Brehm (Schäfers, 2020): Ausgehend vom derzeitigen Grundfreibetrag soll die Knickstelle des Mittelstandsbauchs auf 25.000 Euro zu versteuerndes Einkommen und die Einkommensgrenze des ersten Spitzensteuersatzes auf 80.000 Euro zu versteuerndes Einkommen erhöht werden. Ein erster Reichensteuersatz von $44 \%$ soll ab 150.000 Euro gelten, ein zweiter Reichensteuersatz von $47 \%$ ab 250.000 Euro. Im Gegenzug soll der Solidaritätszuschlag abgeschafft werden. Diese Reform bringt deutliche Entlastungen auch für die Besser- und Hochverdiener. Dadurch entstehen Steuerausfälle von 50 Mrd. Euro im Jahr. Hinzu kommen etwa 2,5 Mrd. Euro durch den Wegfall des Solidari- 
Tabelle 1

\section{Aufkommens- und Verteilungswirkungen von Reformen des Einkommensteuertarifs 2021}

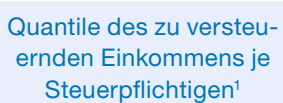
ernden Einkommens je

Steuerpflichtigen 1

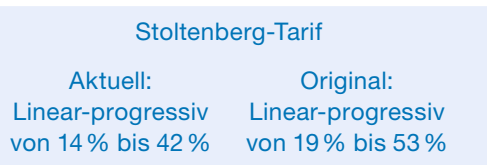

$\begin{array}{cc}\text { DGB Vorschlag } & \text { DIE LINKE } \\ \text { Einkommen- } & \text { Vorschlag } \\ \text { steuertarif } & \text { Einkommen- } \\ & \text { steuertarif }\end{array}$

Güntzler/Brehm (CDU/CSU) Vorschlag Einkommensteuertarif
FDP Vorschlag

Einkommen-

steuertarif

\begin{tabular}{|c|c|c|c|c|c|c|}
\hline \multirow[b]{2}{*}{ 1.-3. Dezil } & \multicolumn{6}{|c|}{ Veränderung Steueraufkommen in Mrd. Euro } \\
\hline & 0,0 & 0,0 & 0,0 & 0,0 & 0,0 & 0,0 \\
\hline 4. Dezil & $-0,2$ & $-0,7$ & $-1,7$ & $-1,8$ & $-0,2$ & $-0,2$ \\
\hline 5. Dezil & $-1,6$ & $-1,2$ & $-3,3$ & $-6,3$ & $-1,5$ & $-1,9$ \\
\hline 6. Dezil & $-3,5$ & $-2,0$ & $-3,6$ & $-8,8$ & $-3,2$ & $-4,4$ \\
\hline 7. Dezil & $-5,4$ & $-2,9$ & $-4,0$ & $-11,4$ & $-4,8$ & $-7,4$ \\
\hline 8. Dezil & $-7,4$ & $-3,8$ & $-4,6$ & $-13,9$ & $-6,8$ & $-11,2$ \\
\hline 9. Dezil & $-9,5$ & $-4,8$ & $-5,2$ & $-16,1$ & $-9,9$ & $-16,9$ \\
\hline 10. Dezil & $-11,5$ & 14,9 & 10,2 & 12,1 & $-23,2$ & $-39,1$ \\
\hline $91 \%-95 \%$ Perzentil & $-5,6$ & $-3,0$ & $-3,1$ & $-8,2$ & $-8,1$ & $-13,6$ \\
\hline $96 \%-99 \%$ Perzentil & $-4,8$ & 2,9 & 1,0 & 0,6 & $-11,3$ & $-17,8$ \\
\hline Top $1 \%$ Perzentil & $-1,1$ & 15,0 & 12,3 & 19,7 & $-3,9$ & $-7,7$ \\
\hline Insgesamt & $-39,1$ & $-0,4$ & $-12,3$ & $-46,2$ & $-49,7$ & $-81,1$ \\
\hline
\end{tabular}

Veränderung Steueraufkommen in Euro je Steuerpflichtigen

\begin{tabular}{|c|c|c|c|c|c|c|}
\hline 1.-3. Dezil & 0 & 0 & 0 & 0 & 0 & 0 \\
\hline 4. Dezil & -30 & -100 & -260 & -274 & -28 & -35 \\
\hline 5. Dezil & -257 & -186 & -527 & -1.000 & -242 & -304 \\
\hline 6. Dezil & -564 & -320 & -579 & -1.424 & -519 & -707 \\
\hline 7. Dezil & -861 & -454 & -638 & -1.808 & -770 & -1.174 \\
\hline 8. Dezil & -1.150 & -589 & -705 & -2.153 & -1.055 & -1.737 \\
\hline 9. Dezil & -1.448 & -740 & -796 & -2.452 & -1.516 & -2.578 \\
\hline 10. Dezil & -1.763 & 2.293 & 1.565 & 1.855 & -3.574 & -6013 \\
\hline $91 \%-95 \%$ Perzentil & -1.684 & -889 & -937 & -2.479 & -2.425 & -4.086 \\
\hline $96 \%-99 \%$ Perzentil & -1.858 & 1.107 & 379 & 232 & -4.373 & -6.931 \\
\hline Top $1 \%$ Perzentil & -1.785 & 24.908 & 20.431 & 32.681 & -6.489 & -12.719 \\
\hline Insgesamt & -598 & -7 & -187 & -707 & -761 & -1.240 \\
\hline
\end{tabular}

Veränderung Steueraufkommen in \% des zu versteuernden Einkommens

\begin{tabular}{|c|c|c|c|c|c|c|}
\hline 1.-3. Dezil & 0,0 & 0,0 & 0,0 & 0,0 & 0,0 & 0,0 \\
\hline 4. Dezil & $-0,2$ & $-0,8$ & $-2,2$ & $-2,3$ & $-0,2$ & $-0,3$ \\
\hline 5. Dezil & $-1,5$ & $-1,1$ & $-3,2$ & $-6,0$ & $-1,5$ & $-1,8$ \\
\hline 6. Dezil & $-2,6$ & $-1,5$ & $-2,7$ & $-6,6$ & $-2,4$ & $-3,3$ \\
\hline 7. Dezil & $-3,2$ & $-1,7$ & $-2,3$ & $-6,6$ & $-2,8$ & $-4,3$ \\
\hline 8. Dezil & $-3,4$ & $-1,8$ & $-2,1$ & $-6,4$ & $-3,1$ & $-5,2$ \\
\hline 9. Dezil & $-3,4$ & $-1,7$ & $-1,9$ & $-5,7$ & $-3,5$ & $-6,0$ \\
\hline 10. Dezil & $-1,9$ & 2,4 & 1,6 & 1,9 & $-3,8$ & $-6,3$ \\
\hline $91 \%-95 \%$ Perzentil & $-2,9$ & $-1,5$ & $-1,6$ & $-4,3$ & $-4,2$ & $-7,1$ \\
\hline $96 \%-99 \%$ Perzentil & $-2,1$ & 1,2 & 0,4 & 0,3 & $-4,9$ & $-7,7$ \\
\hline Top $1 \%$ Perzentil & $-0,5$ & 7,6 & 6,3 & 10,0 & $-2,0$ & $-3,9$ \\
\hline Insgesamt & $-2,4$ & $-0,0$ & $-0,7$ & $-2,8$ & $-3,0$ & $-4,9$ \\
\hline
\end{tabular}

${ }^{1}$ Zusammenveranlagte Paare werden als zwei Steuerpflichtige gezählt. ${ }^{2}$ Festgesetzte Einkommensteuer, nicht veranlagte Lohnsteuer und Abgeltungsteuer, Solidaritätszuschlag auf diese Steuern.

Quelle: Berechnungen mit fortgeschriebenen Daten der Lohn- und Einkommmensteuerstatistik 2016. 
Tabelle 2

Steuerpflichtige Einkommen und Einkommensteuer 2021

\begin{tabular}{|c|c|c|c|c|c|c|c|}
\hline \multirow{2}{*}{$\begin{array}{l}\text { Quantile des zu versteu- } \\
\text { ernden Einkommens je } \\
\text { Steuerpflichtigen }{ }^{1}\end{array}$} & \multirow{2}{*}{$\begin{array}{l}\text { Obergrenze zu versteu- } \\
\text { erndes Einkommen } \\
\text { Euro }\end{array}$} & \multicolumn{2}{|c|}{ Zu versteuerndes Einkommen } & \multicolumn{2}{|c|}{ Einkommensteuer insgesamt ${ }^{2}$} & \multirow[t]{2}{*}{$\begin{array}{l}\text { Durchschnitts- } \\
\text { steuersatz }\end{array}$} & \multirow[t]{2}{*}{$\begin{array}{l}\text { Grenzsteuer- } \\
\text { satz }\end{array}$} \\
\hline & & Mrd. Euro & $\%$ & Mrd. Euro & $\%$ & & \\
\hline 1.-3. Dezil & 9.173 & 49 & 3,0 & 0 & 0,0 & 0,0 & 0,0 \\
\hline 4. Dezil & 13.528 & 78 & 4,7 & 2 & 0,5 & 2,3 & 15,7 \\
\hline 5. Dezil & 18.214 & 105 & 6,3 & 8 & 2,3 & 7,5 & 24,2 \\
\hline 6. Dezil & 23.128 & 133 & 8,1 & 15 & 4,4 & 11,4 & 26,4 \\
\hline 7. Dezil & 28.829 & 171 & 10,3 & 25 & 7,1 & 14,5 & 28,7 \\
\hline 8. Dezil & 35.541 & 218 & 13,2 & 37 & 10,8 & 17,2 & 31,2 \\
\hline 9. Dezil & 47.750 & 280 & 16,9 & 57 & 16,4 & 20,3 & 34,9 \\
\hline 10. Dezil & . & 619 & 37,4 & 204 & 58,6 & 32,9 & 44,6 \\
\hline $91 \%-95 \%$ Perzentil & 65.080 & 191 & 11,6 & 47 & 13,6 & 24,7 & 41,1 \\
\hline $96 \%-99 \%$ Perzentil & 140.496 & 232 & 14,0 & 74 & 21,4 & 32,1 & 46,1 \\
\hline Top $1 \%$ Perzentil & . & 196 & 11,9 & 82 & 23,6 & 41,8 & 46,1 \\
\hline Insgesamt & . & 1.653 & 100,0 & 347 & 100,0 & 21,0 & 34,1 \\
\hline
\end{tabular}

${ }_{1}^{1}$ Zusammenveranlagte Paare werden als zwei Steuerpflichtige gezählt.

Quelle: Berechnungen mit fortgeschriebenen Daten der Lohn- und Einkommmensteuerstatistik 2016.

tätszuschlags auf Abgeltungsteuer und Körperschaftsteuer. In ihrem Wahlprogramm verzichtet die CDU/CSU allerdings auf konkrete Aussagen zum Einkommensteuertarif und kündigt nur allgemein an, kleine und mittlere Einkommen bei der Einkommensteuer entlasten zu wollen (CDU/CSU, 2021). Deutlich offensiver geht die FDP die Reform des Einkommensteuertarifs an (Business Insider, 2021): Ausgehend vom derzeitigen Grundfreibetrag soll bei einem linear-progressiven Tarifverlauf der erste Spitzensteuersatz von $42 \%$ erst bei 90.000 Euro zu versteuerndes Einkommen erreicht und der Solidaritätszuschlag abgeschafft werden. Das bedeutet noch stärkere Entlastungen für die Besser- und Hochverdiener und führt zu Steuerausfällen von $81 \mathrm{Mrd}$. Euro im Jahr, plus die etwa 2,5 Mrd. Euro durch den Wegfall des Solidaritätszuschlags auf Abgeltungsteuer und Körperschaftsteuer.

\section{Potenziale aufkommensneutraler Tarifreformen}

Mit den fortgeschriebenen Daten der Lohn- und Einkommensteuerstatistik lässt sich die Entlastungs- und Belastungsmechanik von Einkommensteuer-Tarifreformen verallgemeinern. Demnach haben die obersten $10 \%$ der Einkommensverteilung $37 \%$ der steuerpflichtigen Einkommen und leisten $59 \%$ des Einkommensteueraufkommens (vgl. Tabelle 2). Die obersten $20 \%$ der Einkommensverteilung erzielen gut $54 \%$ der steuerpflichtigen Einkommen und bringen $75 \%$ des Einkommensteueraufkommens auf. Das bedeutet für eine aufkommensneutrale Reform des Einkommensteuertarifs (vgl. Tabelle 3): Wenn man z. B. die unteren $80 \%$ der Einkommensverteilung um durchschnittlich $1 \%$ des steuerpflichtigen Einkommens entlasten will, muss man die oberen $20 \%$ mit durchschnittlich $0,84 \%$ belasten.
Wenn man bei gleicher Entlastung der unteren $80 \%$ nur die obersten $10 \%$ belasten will, muss man diese um durchschnittlich 1,22\% belasten. Wenn man nur die obersten $5 \%$ belasten will, müssen sie um durchschnittlich $1,76 \%$ belastet werden. Bei der Relation der Grenzbelastungen fallen die Mehrbelastungen der oberen Einkommen etwa doppelt so hoch aus, da hierbei nur die Einkommen berücksichtigt werden, die die jeweilige Einkommensgrenze übersteigen. Daraus ergibt sich für die Steuerpolitik: Wegen der erheblichen Einkommenskonzentration in der Spitze kann man durchaus die unteren Einkommen spürbar entlasten, ohne die hohen Einkommen allzu stark zu belasten. Wobei sich dann aber die Frage stellt, wo hohe Einkommen beginnen. Das $80 \%$-Perzentil-Einkommen, ab dem man zu den einkommensreichsten $20 \%$ der Bevölkerung zählt, liegt bei 35.500 Euro zu versteuerndes Einkommen, das $90 \%$-Perzentileinkommen, ab dem die Top $10 \%$ beginnen, bei 47.800 Euro (vgl. Tabelle 2). Für Alleinstehende mit Standardabzügen entspricht dies Bruttoeinkommen von 44.000 Euro bzw. 59.000 Euro im Jahr, bei höheren Werbungskosten und Sonderausgaben oder Kinderfreibeträgen entsprechend darüber, bei Paaren die doppelten Beträge. Diese sind in der breiten Wahrnehmung keine Spitzenverdiener, sondern eher gehobene Mittelschicht.

Hocheinkommen oder Einkommensreichtum beginnen in der öffentlichen und politischen Wahrnehmung wohl erst bei den obersten $5 \%$. So will etwa die SPD in ihrem Wahlprogramm die obersten $5 \%$ der Einkommensteuerzahler:innen belasten, um die unteren $95 \%$ zu entlasten. Dies entspricht einem zu versteuernden Einkommen von 65.000 Euro im Jahr, bei Standardabzügen bedeutet das ein Bruttoeinkom- 
Tabelle 3

Aufkommensneutrale Belastung höherer Einkommen bei Entlastung niedriger Einkommen um $1 \%$ des zu versteuernden Einkommens

\begin{tabular}{|c|c|c|c|c|c|c|c|}
\hline \multirow[b]{2}{*}{ Belastung ab dem } & \multicolumn{7}{|c|}{ Entlastung bis zum } \\
\hline & 5. Dezil & 6. Dezil & 7. Dezil & 8. Dezil & 9. Dezil & $95 \%$ Perzentil & $99 \%$ Perzentil \\
\hline \multicolumn{8}{|c|}{ Erhöhung der Steuerbelastung in \% des zu versteuernden Einkommens } \\
\hline 6. Dezil & 0,16 & & & & & & \\
\hline 7. Dezil & 0,18 & 0,28 & & & & & \\
\hline 8. Dezil & 0,21 & 0,33 & 0,48 & & & & \\
\hline 9. Dezil & 0,26 & 0,41 & 0,60 & 0,84 & & & \\
\hline 10. Dezil & 0,37 & 0,59 & 0,87 & 1,22 & 1,67 & & \\
\hline $95 \%$ Perzentil & 0,54 & 0,85 & 1,25 & 1,76 & 2,42 & 2,86 & \\
\hline $99 \%$ Perzentil & 1,18 & 1,86 & 2,73 & 3,84 & 5,27 & 6,24 & 7,42 \\
\hline \multicolumn{8}{|c|}{ Erhöhung der Grenzbelastung in \% des zu versteuernden Einkommens } \\
\hline 6. Dezil & 0,28 & & & & & & \\
\hline 7. Dezil & 0,34 & 0,53 & & & & & \\
\hline 8. Dezil & 0,42 & 0,66 & 0,97 & & & & \\
\hline 9. Dezil & 0,53 & 0,84 & 1,23 & 1,73 & & & \\
\hline 10. Dezil & 0,75 & 1,18 & 1,74 & 2,44 & 3,35 & & \\
\hline $95 \%$ Perzentil & 1,05 & 1,65 & 2,42 & 3,41 & 4,67 & 5,54 & \\
\hline $99 \%$ Perzentil & 2,08 & 3,27 & 4,80 & 6,75 & 9,26 & 10,97 & 13,04 \\
\hline
\end{tabular}

Quelle: Berechnungen mit fortgeschriebenen Daten der Lohn- und Einkommmensteuerstatistik 2016.

men von 78.000 Euro, bei Paaren die doppelten Beträge. Das ist schon ein ganz ordentlicher Verdienst. Aber auch der wird wohl überwiegend der oberen Mittelschicht zugerechnet namentlich von den Eliten, die häufig ungleich besser verdienen. Bei den Top $5 \%$ ist die Belastungs- zu Entlastungsrelation deutlich ungünstiger: Ihre Durchschnittsbelastung muss man fast um die dreifachen Prozentpunkte erhöhen, um die man die Belastung der unteren 95\% reduziert. Die Grenzbelastungen müssen um 5,5 Prozentpunkte steigen. Dies ist vor allem für die intramarginalen Anreize relevant, also mehr zu arbeiten, zu investieren oder Steuergestaltungen zu nutzen - Letzteres spielt bei den oberen Einkommensgruppen eine größere Rolle, da diese oft mehr Flexibilität bei den wirtschaftlichen Betätigungen haben. Dabei ist auch zu berücksichtigen: Vor allem die sehr hohen Unternehmens- und Vermögenseinkommen unterliegen häufig nicht mehr der progressiven Einkommensteuer, soweit sie in Unternehmen, Holdinggesellschaften oder Stiftungen thesauriert werden. Dies führt zu Effektivbelastungen mit Unternehmensteuern von $20 \%$ bis $30 \%$, mitunter auch deutlich niedriger, wenn Auslandsgewinne erzielt oder Steuergestaltungen genutzt werden (Bach, Beznoska und Steiner, 2016). Erst wenn die Gewinne in die Privatsphäre ausgeschüttet werden, was aber nur selten geschieht, fällt darauf zusätzlich Abgeltungsteuer an. Damit werden diese Einkommen häufig niedriger besteuert als die Erwerbseinkommen der oberen Mittelschichten und Besserverdienenden - zumal, wenn man die Sozialbeiträge mitrechnet. Die sehr hohen Unternehmens- und Vermögenseinkommen transparent in der persönlichen Einkommensteuer zu erfassen, ist zwar möglich, aber steuertechnisch und politisch schwierig. Grundsätzlich gab es das in Deutschland bis 2001 mit der weitgehenden Integration der Körperschaftsteuer in die Einkommensteuer - durch das Vollanrechnungsverfahren und den hohen Körperschaftsteuersatz für einbehaltene Gewinne. Dies wurde aufgebeben angesichts der Globalisierung der Finanzmärkte, internationalem Steuersenkungswettlauf bei Unternehmen- und Kapitaleinkommensteuern sowie europarechtlichen Vorbehalten. Die Rückkehr zu einem solchen System würde intensive internationale Abstimmungen erfordern.

Als zweitbeste Lösung könnte eine Vermögensteuer auf sehr hohe Vermögen erhoben werden. Dafür gibt es ein beträchtliches Aufkommenspotenzial: Eine Wiedererhebung der Vermögensteuer mit einem persönlichen Freibetrag von 2 Mio. Euro und einem Steuersatz von 1\% könnte ein Aufkommen von schätzungsweise 24 Mrd. Euro im Jahr erzielen (Bach, 2020): Bei einem zusätzlichen Freibetrag für Betriebsvermögen und Unternehmensbeteiligungen von 5 Mio. Euro läge das Steueraufkommen noch bei 17,5 Mrd. Euro im Jahr. Selbst eine Superreichen-Vermögensteuer von $1 \%$ auf persönliche Vermögen über 20 Mio. Euro könnte noch ein jährliches Aufkommen von 9,5 Mrd. Euro erzielen. Auch eine reformierte Erbschaftsteuer könnte längerfristig ein ähnliches Aufkommen erzielen, wenn Übertragungen von hohen Unternehmensvermögen nicht mehr weitgehend steuerbefreit werden. Dieses 
Aufkommenspotenzial ließe sich in eine Reform zur Entlastung der unteren und mittleren Einkommen einbeziehen.

Über die betrachteten unmittelbaren Erstrundeneffekte hinaus sind wirtschaftliche Wirkungen zu berücksichtigen, die auch das Aufkommen betreffen. Kurz- und mittelfristig können die Steuerpflichtigen bei Arbeitsangebot, Investitionen oder Steuergestaltungen reagieren, längerfristig auch bei Bildung und Weiterbildung, Berufswahl und Karriere (Doerrenberg, Peichl und Siegloch, 2017). Bei den skizzierten aufkommensneutralen Reformen dürften negative Reaktionen der stärker belasteten Steuerpflichtigen mit hohen Einkommen (oder Vermögen) die positiven Reaktionen der entlasteten Steuerpflichtigen mit geringen und mittleren Einkommen tendenziell übersteigen. Denn Hocheinkommensbezieher reagieren bei Investitionen oder Steuergestaltungen vermutlich stärker als Niedrigeinkommensbezieher und Mittelschichten beim Arbeitsangebot. Zudem steigen die Durchschnitts- und vor allem die Grenzbelastungen bei ersteren stärker, als sie bei letzteren sinken. Bei Unternehmenseinkommen, die einen großen Anteil der hohen steuerpflichtigen Einkommen ausmachen, kann die Thesaurierungsbegünstigung nach § 34a EStG oder die neue Option zur Körperschaftsteuer genutzt werden, um für die einbehaltenen Gewinne die Erhöhung der Spitzensteuersätze zu vermeiden. Dem wirken Nachfrageeffekte auf den privaten Verbrauch entgegen, da die entlasteten unteren und mittleren Einkommen stärker konsumieren dürften als die belasteten Hocheinkommensbezieher:innen. Bei der Investitionsnachfrage geht es in die andere Richtung. ${ }^{1}$ Insgesamt sind die längerfristigen wirtschaftlichen Wirkungen der Reformen also unbestimmt und wohl tendenziell schwach negativ, sie hängen von den jeweiligen Szenarien und auch der gesamtwirtschaftlichen Konstellation ab.

\section{Fazit}

Der schnelle Anstieg der Progression des Einkommensteuertarifs belastet Mittelschichten und Besserverdiener relativ stark. Wenn man dies durch eine Anhebung des Grundfreibetrags oder eine Abmagerung des Mittelstandsbauchs mindern will, entstehen leicht Steuerausfälle von höheren

1 Gesamtwirtschaftliche Wachstumswirkungen von Steuerentlastungen werden meist geringer eingeschätzt als Erhöhungen der staatlichen Investitionsausgaben, Gechert (2015); Gechert und Heimberger (2021). zweistelligen Milliardenbeträgen im Jahr. Erhöhungen der Spitzensteuersätze wirken dem entgegen, allerdings sind die Aufkommenspotenziale bei den Hochverdienern begrenzt. Wenn z. B. die unteren $95 \%$ der Bevölkerung um $1 \%$ ihres steuerpflichtigen Einkommens entlastet werden sollen, muss man die obersten $5 \%$ mit knapp 3\% belasten, die Grenzbelastungen müssen um 5,5 Prozentpunkte steigen. Hohe Unternehmens- und Vermögenseinkommen unterliegen häufig nicht mehr der progressiven Einkommensteuer, soweit sie in Kapitalgesellschaften erzielt werden. Diese könnten mittelbar durch Vermögensteuern belastet werden.

\section{Literatur}

Bach, S. (2020), Vermögensabgabe DIE LINKE. Aufkommen und Verteilungswirkungen, Forschungsprojekt im Auftrag der Fraktion DIE LINKE im Bundestag und der Rosa-Luxemburg-Stiftung, DIW Berlin Politikberatung kompakt, 157, 43.

Bach, S., M. Beznoska und V. Steiner (2016), Wer trägt die Steuerlast in Deutschland? Steuerbelastung nur schwach progressiv, DIW Wochenbericht, 51, 52.

Bach, S. und H. Buslei (2017), Wie können mittlere Einkommen beim Einkommensteuertarif entlastet werden?, DIW Wochenbericht, 20.2017.

Beznoska, M. und T. Hentze (2021), Wahlprogramme zur Einkommensteuer: Unten entlasten, oben belasten, IW-Kurzbericht, 22/2021.

Buggeln, M. (2018), Die Debatten um staatliche Zugriffsmöglichkeiten auf den privaten Reichtum. Der Einkommensteuerspitzensatz in Deutschland 1871-1955, Vierteljahrschrift für Sozial- und Wirtschaftsgeschichte, 105, 337-364.

CDU/CSU (2021), Das Programm für Stabilität und Erneuerung. Gemeinsam für ein modernes Deutschland, 34 und 71.

Business Insider (2021), 14. April 2021.

Deutscher Gewerkschaftsbund (DGB) (2021), Steuerpolitisches Gesamtkonzept. Position des Deutschen Gewerkschaftsbundes, 30. März.

Doerrenberg, P., A. Peichl und S. Siegloch (2017), The elasticity of taxable income in the presence of deduction possibilities, Journal of Public Economics, 151, 41-45.

Esser, C. (2009), Die Belastung durch Einkommensteuer und Sozialabgaben. Entwicklung und Perspektiven, IFSt-Schrift, 454, 65.

Gechert, S. (2015), What fiscal policy is most effective? A meta-regression analysis, Oxford Economic Papers, 67, 553.

Gechert, S. und P. Heimberger (2021), Do corporate tax cuts boost economic growth?, IMK Working Paper, 210.

OECD (2021), Taxing Wages 202.

Pollak, H. (1980), Steuertarife, Handbuch der Finanzwissenschaft, Bd. 2, 3. Aufl., 239-266.

Popitz, J. (1926), Einkommensteuer, in Handwörterbuch der Staatswissenschaften, 3. Bd., 400-491.

Schäfers, M. (2020), Rütteln am Steuerdogma, Frankfurter Allgemeine Zeitung, 6. Februar.

Schumpeter, J. (1929), Ökonomie und Soziologie der Einkommensteuer, Der deutsche Volkswirt, IV, 382.

Statistisches Bundesamt (2020), Lohn- und Einkommensteuer - Fachserie 14 , Reihe $7.1-2016$

Title: Current Reform Proposals for the German Personal Income Tax Schedule: Relieve the Middle Class and High Earners, Burden High Incomes

Abstract: The current German personal income tax schedule imposes a relatively heavy burden on the middle class due to the rapid rise in tax progression. Relief through an increase in the basic tax-free allowance or a flattening of the steep increase in marginal tax rates can easily lead to tax losses upwards of double-digit billions per year. Top tax rates reduce these losses, but the revenue potential from high earners is limited. For example, if the bottom $95 \%$ of the population is to be relieved of $1 \%$ of their taxable income, the top $5 \%$ have to be charged almost $3 \%$, the marginal tax rate must increase by 5.5 percentage points.

JEL Classification: $H 24, H 22, D 31$ 